\title{
Development of a Foresight Incentive Algorithm and Its Application in Construction Conflicts
}

\author{
Zhongfu Qin ${ }^{1}$, Fang $\mathrm{Hua}^{2}$, Haizhen Wen ${ }^{* 3}$ and Bailu $\mathrm{Cao}^{4}$ \\ ${ }^{1}$ Associate Professor, Institute of Civil Engineering Management, Zhejiang University, China \\ ${ }^{2}$ Engineer, China State Construction Engineering (Hong Kong) Ltd. \\ ${ }^{3}$ Associate Professor, Institute of Civil Engineering Management, Zhejiang University, China \\ ${ }^{4}$ Graduate Student, Institute of Civil Engineering Management, Zhejiang University, China
}

\begin{abstract}
There often exist multiple equilibria in the algorithm results of metagame theory and conflict analysis, and some of the equilibria may be non-Pareto optimal or low stable. Thus, it is still a problem for decisionmakers to choose among the equilibria. On the basis of the traditional metagame algorithm, this paper develops a new algorithm by taking "foresight incentive" into consideration, so as to obtain more terse and stable equilibria. The analysis steps of the foresight incentive algorithm include generating a route for each player in each scenario, identifying the check-point in each route, examining if each check-point is foresight incentive point, and analyzing the stability of each scenario for each player. Finally, through the case study of a real construction conflict, the effectiveness and superiority of the foresight incentive algorithm are verified.
\end{abstract}

Keywords: foresight incentive algorithm; route; foresight incentive point; conflict; construction

\section{Introduction}

Conflicts on interests and aims among each party in the field of construction engineering are unavoidable, because of the diversity of crafts as well as the complexity of organization. The main idea of metagame theory, brought forth by Howard [1971] $]^{1)}$, is to consider each player's options as a function, and select options based on considering other players' possible reactions, so as to achieve common stable solutions for all parties involved in the conflicts. Based on metagame theory, Fraser \& Hipel [1984] $]^{2}$ have put forward conflict analysis, which develops several new concepts and methods, and overcomes some flaws in metagame analysis. In addition, Kilgour $[1987]^{3)}$ introduced the Graph Model theory, in which the scenarios, instead of players' options, are the basic unit, making the analysis of conflicts more realistic. To permit practitioners to conveniently apply the graph model for conflict resolution to practical real-world conflict problems, Fang et al. [1993] $]^{4)}$ developed a decision support system of GMCR (Graph Model for Conflict Resolution), by considering the irreversible and common moves route in the process of scenario transitions. The effectiveness of these theoretical achievements has been verified through a number of practical conflict scenarios. ${ }^{5-10)}$

\footnotetext{
*Contact Author: Haizhen Wen, Associate Professor,

Institute of Civil Engineering Management, Zhejiang University, Hangzhou, China, 310058

Tel: +86158 88826824 Fax: +86 57188208685

E-mail: wenhaizhen@263.net
}

(Received April 18, 2011 ; accepted July 24, 2012)
However, there often exist multiple equilibria in the analysis results of metagame theory or conflict algorithm, especially when considering the irreversible and common moves route. Meanwhile, some of the equilibria may be non-Pareto optimal or low stable. Thus, it is still a problem for decision-makers to choose from among these equilibria. However, it does exist in the real world that a player in a certain scenario may take the option that is more favorable for him with farsight, while being adverse for him with short sight. In this study, this kind of situation is defined as "foresight incentive". In order to maximize their utility, all the parties in the construction conflicts often rack their brains to guess each other's attempts, and make use of every strategy. It is possible that the foresight incentive factors would be taken into consideration, for those smart players who have enough foresight. On the basis of traditional conflict analysis, this paper will develop a foresight incentive algorithm, so as to obtain more stable, and more far-sighted equilibria, which therefore may be more easily accepted by the parties involved in the conflicts.

\section{The Idea of Foresight Incentive Algorithm \\ 2.1 The proposal of foresight incentive algorithm}

In the assessment of the stability of a specific scenario for a given player, there are three conditions. First, the player has no unilateral movement (UM) from the scenario. Second, the player has unilateral movements, but he cannot move to any more favorable scenario by his first unilateral movement. The second condition is defined as unilateral disimprovement $(U D)$. Finally, the player can move to more favorable 
scenarios by his first unilateral movement, which is defined as unilateral improvement $(U I)$. For traditional metagame and conflict analysis theory, the scenarios in the first and second conditions are considered as stable for the player. However, imagine this type of two-player game: player 1 is in scenario $a$, for whom scenario $a$ is more favorable than scenario $b$, and scenario $c$ is more favorable than scenario $a$. Player 1 can move to scenario $b$ but cannot move to scenario $c$ by his $U M$. For player 2 scenario $c$ is more favorable than scenario $b$, meanwhile player 2 can move from $b$ to $c$ by his $U M$. In this case, if player 1 first moves to $b$ from $a$ intentionally, and then expects player 2 's movement from $b$ to $c$, he will finally reach the scenario that is more favorable for him and unavailable directly by himself. This situation is defined as foresight incentive. With the concept of foresight incentive, some of the less stable equilibria obtained by traditional metagame algorithm will be removed, and the equilibria obtained by the new algorithm (called "foresight incentive algorithm", see below) will be more terse and stable.

\subsection{The stability analysis of foresight incentive algorithm}

If player 1 has no $U M$ from scenario $a$, then $a$ is stable for him. Suppose that player 1 can move from $a$ to $b$ by $U M$, and be sure that he can finally reach scenario $z$, which is more favorable than $a$ through a series of alternate $U M S$. These alternate $U M S$ need player 2's co-operation, which has the precondition that $z$ is also more favorable than $b$ for player 2. In this case, player 1 will move from $a$ to $b$ out of the foresight incentive, and expect that he can finally reach $z$ through a series of alternate UMs. Therefore, $a$ is unstable for player 1 . Here the movement of player 1 from $a$ to $b$ is defined as the foresight incentive route. If player 1 has no foresight incentive route from scenario $a$, then $a$ is stable for him. If a particular scenario is stable for all players, it is a globally stable scenario, i.e., an equilibrium.

\section{Basic Assumptions of Foresight Incentive Algorithm \\ 3.1 Players}

A non-cooperative game with two DMs is studied in this paper. Generally, all players in game theory are supposed to be rational. In addition, suppose that all the players in this study have the foresight for the changes of the conflicts, and are willing to move to their most favorable scenarios, meanwhile accepting temporary adversity.

\subsection{Feasible scenarios}

Suppose that all the players' options total to $m$. There will be $2^{m}$ scenarios mathematically. However, considering the concrete conditions of conflicts in practice, some of the scenarios may be infeasible physically, logically, or reasonably. Excluding all these infeasible scenarios, the set of feasible scenarios will be obtained. The following analysis will be conducted in the set of feasible scenarios.

\subsection{The way of players' movement}

Suppose that a player can move from scenario $a$ to scenario $b$ by $U M$, and subsequently moves from scenario $b$ to scenario $c$ by $U M$. That is to say, the player has at least two UMs in scenario $a$, from $a$ to $b$ and from $a$ to $c$, respectively. In this study, if a player has more than one $U M$ in a scenario, including the original scenario, each route generated by each $U M$ will be analyzed. In addition, only the case of alternate $U M s$ by both players will be taken into account, while the case of one player's continuous $U M S$ will not be considered.

\section{Developing the Foresight Incentive Algorithm 4.1 Generating the movement routes}

As stated above, in the assessment of the stability of scenario $a$ for player 1 , one needs to know that if player 1 can reach scenario $z$, which is more favorable for both players from scenario $a$ through a series of alternate $U M s$. Therefore, first one needs to generate all the possible routes along which player 1 can move from scenario $a$ to scenario $z$ through a series of alternate $U M S$. Second, one determines if each route is feasible for both players.

If one player can move from scenario $S_{0}$ to scenario $S_{x}$ through a series of both players' alternate $U M s$, the chain consisting of these scenarios and alternate $U M S$ is called "Route", denoted as $R$, as follows:

\section{$\mathrm{R}=\left\{S_{0}, S_{1}, S_{2}, \cdots, S_{x-1}, S_{x}\right\}$}

In the process of players' alternate $U M S$, when player 1 moves to scenario $S_{x}$, there may be several cases for player 2 as shown in Table 1., where the set of scenarios which player 2 can reach from scenario $S_{x}$ is denoted as $m_{2}\left(s_{x}\right)$, and the number of elements of the set is denoted as $N_{2}{ }^{U M}\left(S_{x}\right)$.

All the cases listed in Table 1. are discussed specifically as follows.

Case 1: Player 2 has no $U M$ in scenario $S_{x}$. This scenario is an end of the route, which is defined as "original end", in order to distinguish it from other types of ends in this paper.

Case 2: Player 2 has only one $U M$ in scenario $S_{x}$, while the scenario reached by this $U M$ is one of the scenarios of the route $\left\{S_{0}, S_{1}, \cdots, S_{x}\right\}$. Then it will form a circle, and may lead to an infinite loop, which is a waste of time and cost in an actual conflict. In this study, players are considered to be "smart enough" to avoid forming circles at any time. That is to say, player 2 will give up those $U M s$ that can lead to a circle. As player 2 only has one $U M$ in scenario $S_{x}$, player 2 will stop there. Therefore, scenario $S_{x}$ is called a "derived end" of the route.

Case 3: Player 2 has only one $U M$ in scenario $S_{x}$, and the scenario reached by this $U M$ is not any one of the scenarios of the route $\left\{S_{0}, S_{1}, \cdots, S_{x}\right\}$. Then both players will continue the alternate $U M S$, until one of 
Table 1. The Possible Cases in Route Generation

\begin{tabular}{c|l|l}
\hline Case & Condition set \\
\hline Case 1 & $N_{2}{ }^{U M}\left(S_{x}\right)=0$ & $m_{2}\left(s_{x}\right) \in R$ \\
Case 2 & \multirow{2}{*}{$N_{2}{ }^{U M}\left(S_{x}\right)=1$} & $m_{2}\left(s_{x}\right) \notin R$ \\
\hline Case 3 & & $\forall m_{2}\left(s_{x}\right): m_{2}\left(s_{x}\right) \in R$ \\
Case 4 & \multirow{3}{*}{$N_{2}^{U M}\left(S_{x}\right) \geq 2$} & $\forall m_{2}\left(s_{x}\right): m_{2}\left(s_{x}\right) \notin R$ \\
Case 5 & & $\exists m_{2}\left(s_{x}\right): m_{2}\left(s_{x}\right) \in R, \exists m_{2}\left(s_{x}\right): m_{2}\left(s_{x}\right) \notin R$ \\
\hline Case 6 & &
\end{tabular}

them reaches the original end or derived end.

Case 4: Player 2 has no less than two UMs in scenario $S_{x}$, and each one of these $U M s$ will lead to a circle. Then the player will stop in scenario $S_{x}$, which is called a "derived end" of the route.

Case 5: Player 2 has no less than two UMs in scenario $S_{x}$, and each one of these $U M S$ will not lead to a circle. Then there will be $N_{2}{ }^{U M}\left(S_{x}\right)$ branches diverging from scenario $S_{x}$. In each branch, both players will continue the alternate $U M S$, until one of them reaches the original end or derived end.

Case 6: Player 2 has no less than two $U M S$ in scenario $S_{x}$, and some of the $U M S$ will lead to a circle, while some will not. Then the number of branches diverging from scenario $S_{x}$ is the number of the $U M S$ which will not form a circle. Similarly, in each branch, both players will continue the alternate $U M S$, until one of them reaches the original end or derived end.

After reaching an end, no matter whether it is an original end or a derived end, one of the players can not move any more. Therefore, both players' alternate $U M s$ will stop at that end. The chain formed by both players' alternate $U M s$ from the original scenario $S_{0}$ to the terminal scenario $S_{t}$ is called route $R$. All the scenarios in route $R$ can be arranged in the order reached, as follows, so as to simplify the description of examining routes:

$$
R=S_{0} S_{1} S_{2} \cdots S_{t-1} S_{t}
$$

\subsection{Setting check-points}

The generation of routes is the first step in analyzing the stability of a scenario. Then, in each route, one needs to identify which scenario can be regarded as a check-point, and what will be used to examine if the foresight incentive point exists in the route.

As stated above, both players are considered to be rational and far-sighted. Therefore, if the end $S_{t}$ of route $R$ is more favorable than scenario $S_{t-1}$ for player 1 , he will certainly move from $S_{t-1}$ to $S_{t}$, for he knows that player 2 cannot move any more from scenario $S_{t}$. Here scenario $S_{t}$ is called the "check-point" of route $R$. If the end $S_{t}$ of route $R$ is not more favorable than scenario $S_{t-1}$ for player 1 , he will certainly not move from $S_{t-1}$ to $S_{t}$, for he knows that player 2 cannot move any more from scenario $S_{t}$. Here scenario $S_{t-1}$ is the end of route $R$ instead of scenario $S_{t}$. Further, after the end of route $R$ becomes scenario $S_{t-1}$, a similar discussion can be conducted for player 2 . If the end $S_{t-1}$ of route $R$ is more favorable than scenario $S_{t-2}$ for player 2 , he will certainly move from $S_{t-2}$ to $S_{t-1}$, for he knows that player 1 cannot move any more from scenario $S_{t-1}$. Here scenario $S_{t-1}$ is called the "check-point" of route $R$. If the end $S_{t-1}$ of route $R$ is not more favorable than scenario $S_{t-2}$ for player 2, he will certainly not move from $S_{t-2}$ to $S_{t-1}$, for he knows that player 1 cannot move any more from scenario $S_{t-1}$. Here scenario $S_{t-2}$ is the end of route $R$ instead of scenario $S_{t-1}$. And so on, the discussion can be continued until a "check-point" in route $R$ appears, which is shown in Table 2. If the check-point is the original scenario of route $R$, there is not any foresight incentive point in the route. If there is a "check-point" in route $R$, one needs to verify if the check-point is the foresight incentive point in the route.

\subsection{Examination of foresight incentive points}

Suppose that scenario $S_{t}$ is the check-point of route $R$. For player 1 in scenario $S_{t-1}$, scenario $S_{t}$ is more favorable than scenario $S_{t-1}$, meanwhile he knows that player 2 cannot move any more from scenario $S_{t}$, so player 1 will certainly move from scenario $S_{t-1}$ to scenario $S_{t}$. In this case, player 2 in scenario $S_{t-2}$ also knows very well that player 1 will move from scenario $S_{t-1}$ to scenario $S_{t}$. Therefore, the only condition for player 2 to move from scenario $S_{t-2}$ to scenario $S_{t-1}$ is $S_{t} \succ_{2} S_{t-2}$. Suppose that the condition is satisfied, and player 2 chooses to move from scenario $S_{t-2}$. Similarly, the only condition for player 1 to move from scenario $S_{t-3}$ is $S_{t \succ 1} S_{t-3}$. And so on, if player 1 is willing to move from scenario $S_{0}$ and stop in scenario $S_{t}$ through a series of both players' alternate $U M S$, scenario $S_{t}$ needs to meet the following conditions:

$$
\left\{\begin{array}{l}
S_{t} \succ_{1} S_{0}, S_{t} \succ_{1} S_{2}, S_{t} \succ_{1} S_{4}, \ldots, S_{t} \succ_{1} S_{2 y} \\
S_{t} \succ_{2} S_{1}, S_{t} \succ_{2} S_{3}, S_{t} \succ_{2} S_{5}, \ldots, S_{t} \succ_{2} S_{2 y+(-1)^{t}}
\end{array}\right.
$$

where $y=[(t-1) / 2]$, and represents the integers of $(t$ $-1) / 2, t \geq 2$.

If scenario $S_{t}$ meets the above conditions, it is called the "foresight incentive point" of route $R$, and route $R$ is called the "foresight incentive route". If one of the routes starting from scenario $S_{0}$ for player 1 is a foresight incentive route, player 1 will move from the original scenario $S_{0}$ out of the foresight incentive. Therefore, scenario $S_{0}$ is unstable for player 1 . If each route starting from scenario $S_{0}$ for player 1 is not foresight incentive route, scenario $S_{0}$ is stable for player 1 . 


\begin{tabular}{c|c}
\hline Condition set & Check-points \\
\hline$S_{t} \succ_{1} S_{t-1}$ & $S_{t}$ \\
\hline$S_{t} \prec_{1} S_{t-1}, S_{t-1} \succ_{2} S_{t-2}$ & $S_{t-1}$ \\
\hline$S_{t} \prec_{1} S_{t-1}, S_{t-1} \prec_{2} S_{t-2}, S_{t-2} \succ_{1} S_{t-3}$ & $S_{t-2}$ \\
\hline$\cdots \cdots$ & $\cdots$ \\
\hline$S_{t} \prec_{1} S_{t-1}, S_{t-1} \prec_{2} S_{t-2}, S_{t-2} \prec_{1} S_{t-3}, \cdots, S_{2} \prec_{2} S_{1}, S_{1} \succ_{1} S_{0}$ & $S_{1}$ \\
\hline$S_{t} \prec_{1} S_{t-1}, S_{t-1} \prec_{2} S_{t-2}, S_{t-2} \prec_{1} S_{t-3}, \cdots, S_{2} \prec_{2} S_{1}, S_{1} \prec_{1} S_{0}$ & $S_{0}$ \\
\hline
\end{tabular}

\section{Case Study}

\subsection{Case illustration}

The Xin'ao Group, the developer and the one who had the housing property rights, rented out its shop at No. 5-25, Anding Road, Chaoyang District, Beijing to the Oriental Kaisheng Trading Company in late 2007. In July 2008, the Oriental Kaisheng Trading Company subleased part of its property to Mrs. Qin Rong to run a restaurant for three years. In August 2009, Qin Rong learned that the house would be removed, so she stopped paying rent. In September 2009, the Oriental Kaisheng Trading Company submitted a case to the Chaoyang Court to terminate the house lease contract for Qin Rong's defaults on her rent. In late November 2009, the Oriental Kaisheng Trading Company cut off the house's water and electricity supply and repeatedly tried to seal the restaurant. In early December 2009, Mrs. Qin recruited a full-time "nail household" to guard the restaurant through the website (http://blog. sina.com.cn/u/1669141710). Mr. Lu applied to guard the restaurant, and $\mathrm{Mr}$. $\mathrm{Xu}$ applied in charge of the petition and litigation issues.

Mrs. Qin refused to move on the grounds that the lessor must compensate the renter when the house lease contract is terminated due to natural disasters or requisition, according to the contract. However, the Xin'ao Group claimed that the renter has to move unconditionally and no compensation was needed when the demolition occurred, also according to the contract. The two sides failed to reach a consensus for a long time and it was difficult for the court to decide.

\subsection{Conflict modeling}

\subsubsection{Setting players}

According to the aforementioned case, the parties involved in this demolition conflict mainly focused on the amount of compensation. The two main players, who have antagonistic interests, are the developer, the Xin'ao Group and Oriental Kaisheng Trading Company, who promoted the demolition process, and the relocation household, Mrs. Qin, Mr. Lu and Mr. $\mathrm{Xu}$, who hampered the demolition process.

\subsubsection{Setting options and feasible scenarios}

In this study, the developer (player 1) is considered to have three options as follows:

(1) Compensation, that is to say, supply enough compensation to meet the relocation household's requirement.
(2) Pressure, that is to say, stop water and electricity supply, and seal the shop.

(3) Removal, that is to say, carry out mandatory removal.

The relocation household (player 2) also has three options:

(1) Petition, which means that $\mathrm{Mr}$. Xu continues taking petitions.

(2) Litigation, which means taking legal action if the Xin'ao Group carries out mandatory removal.

(3) Guard, which means that Mr. Lu guards the house.

Since there are six options in total, the number of scenarios is $2^{6}=64$. After the infeasible scenarios are removed, the remaining twelve feasible scenarios are listed as shown in Table 3.

\subsubsection{Setting preference vectors}

The preference vector for each player is shown in Table 4. The smaller the figure is, the more favorable the scenario is for the player.

\subsection{Stability analysis}

Both conflict analysis and foresight incentive algorithm developed in this study are applied to this practical conflict for stability analysis (see Hua $[2010]^{12)}$ ), and the results are shown in Table 5 . Then the results obtained by these two methods are compared.

\subsubsection{Stability analysis based on conflict analysis}

The equilibria gained by conflict analysis are scenarios 5, 20, and 42. Scenario 5 represents that the developer supplying enough compensation to meet the relocation household's requirements and carries out removal, while the relocation household just moves away with no other action. Scenario 20 means that the developer carries out mandatory removal without any compensation, while the relocation household does not guard the house, but takes legal action. Scenario 42 means that the relocation household guards the house and continues taking petitions, while the developer puts pressure on the relocation household. Here $5 \succ_{1} 42 \succ_{1}$ 20, and $5 \succ_{2} 42 \succ_{2} 20$. That is to say, scenario 5 is Pareto superior to scenario 42, and scenario 42 is Pareto superior to scenario 20. This shows that there are three equilibria gained by the traditional stability analysis method, and two of them are non-Pareto optimal solutions. 
Table 3. Feasible Scenarios

\begin{tabular}{|c|c|c|c|c|c|c|c|c|c|c|c|c|c|}
\hline Players & Options & \multicolumn{12}{|c|}{ Feasible scenarios } \\
\hline \multirow{3}{*}{$\begin{array}{c}\text { Developer } \\
\text { (player 1) }\end{array}$} & Compensation & 0 & 1 & 0 & 0 & 0 & 0 & 0 & 0 & 0 & 0 & 0 & 0 \\
\hline & Pressure & 0 & 0 & 0 & 0 & 0 & 0 & 1 & 0 & 0 & 1 & 0 & 0 \\
\hline & Removal & 0 & 1 & 0 & 1 & 1 & 0 & 0 & 1 & 0 & 0 & 1 & 1 \\
\hline \multirow{3}{*}{$\begin{array}{l}\text { Relocation } \\
\text { household } \\
\text { (player 2) }\end{array}$} & Petition & 0 & 0 & 1 & 1 & 0 & 0 & 0 & 0 & 1 & 1 & 1 & 0 \\
\hline & Litigation & 0 & 0 & 0 & 0 & 1 & 0 & 0 & 0 & 0 & 0 & 0 & 1 \\
\hline & Guard & 0 & 0 & 0 & 0 & 0 & 1 & 1 & 1 & 1 & 1 & 1 & 1 \\
\hline \multicolumn{2}{|c|}{ Decimal Number } & 0 & 5 & 8 & 12 & 20 & 32 & 34 & 36 & 40 & 42 & 44 & 52 \\
\hline
\end{tabular}

Table 4. The Preference Vectors of Players

\begin{tabular}{c|c|c|c|c|c|c|c|c|c|c|c|c|c|c|c|c|}
\hline \multicolumn{2}{c|}{ Feasible scenarios } & 0 & 5 & 8 & 12 & 20 & 32 & 34 & 36 & 40 & 42 & 44 & 52 \\
\hline \multirow{2}{*}{ Preference vectors } & Developer & 9 & 2 & 10 & 1 & 7 & 11 & 3 & 5 & 12 & 4 & 6 & 8 \\
\cline { 2 - 22 } & $\begin{array}{c}\text { Relocation } \\
\text { household }\end{array}$ & 5 & 1 & 4 & 12 & 8 & 3 & 7 & 11 & 2 & 6 \\
\hline
\end{tabular}

Table 5. The Results of Stability Analysis Based on Conflict Analysis and Foresight Incentive Algorithm

\begin{tabular}{|c|c|c|c|c|c|c|c|c|c|c|c|c|c|}
\hline \multicolumn{2}{|c|}{ Feasible scenarios } & 0 & 5 & 8 & 12 & 20 & 32 & 34 & 36 & 40 & 42 & 44 & 52 \\
\hline \multirow{3}{*}{ Conflict analysis } & Developer & $\mathrm{u}$ & $\mathrm{r}$ & $\mathrm{u}$ & $\mathrm{r}$ & $\mathrm{r}$ & $\mathrm{u}$ & $\mathrm{r}$ & $\mathrm{u}$ & $\mathrm{u}$ & $\mathrm{r}$ & $\mathrm{u}$ & $\mathrm{r}$ \\
\hline & $\begin{array}{l}\text { Relocation } \\
\text { household }\end{array}$ & $\mathrm{r}$ & $\mathrm{r}$ & $\mathrm{S}$ & $\mathrm{u}$ & $\mathrm{r}$ & $\mathrm{S}$ & $\mathrm{u}$ & $\mathrm{u}$ & $\mathrm{r}$ & $\mathrm{r}$ & $\mathrm{u}$ & $\mathrm{u}$ \\
\hline & Equilibrium & $\times$ & $\mathrm{E}$ & $\times$ & $\times$ & $\mathrm{E}$ & $x$ & $\times$ & $\times$ & $\times$ & $\mathrm{E}$ & $\times$ & $\times$ \\
\hline \multirow{3}{*}{$\begin{array}{c}\text { Foresight incentive } \\
\text { algorithm }\end{array}$} & Developer & $\mathrm{u}$ & $\mathrm{r}$ & $\mathrm{u}$ & $\mathrm{r}$ & $\mathrm{r}$ & $\mathrm{u}$ & $\mathrm{u}$ & $\mathrm{u}$ & $\mathrm{u}$ & $\mathrm{u}$ & $\mathrm{u}$ & $\mathrm{r}$ \\
\hline & $\begin{array}{l}\text { Relocation } \\
\text { household }\end{array}$ & $\mathrm{r}$ & $\mathrm{r}$ & $\mathrm{u}$ & $\mathrm{u}$ & $\mathrm{u}$ & $\mathrm{u}$ & $\mathrm{u}$ & $\mathrm{u}$ & $\mathrm{u}$ & $\mathrm{u}$ & $\mathrm{u}$ & $\mathrm{u}$ \\
\hline & Equilibrium & $x$ & $\mathrm{E}$ & $x$ & $\times$ & $x$ & $x$ & $\times$ & $x$ & $x$ & $\times$ & $\times$ & $x$ \\
\hline
\end{tabular}

\subsubsection{Stability analysis based on foresight incentive algorithm}

Take the route $42 \rightarrow 40 \rightarrow 0 \rightarrow 5$ as an example. According to section 4.1, there is no $U M$ from scenario 5 for player 2, so scenario 5 is the original end. As $5 \succ_{1}$ 0 , scenario 5 is the check-point according to section 4.2. Furthermore, as $5 \succ_{1} 0,5 \succ_{1} 42$, and $5 \succ_{2} 40$, scenario 5 is the foresight incentive point and the route is foresight incentive route according to section 4.3. Therefore, scenario 42 is unstable for player 1 .

Take the route $20 \rightarrow 12 \rightarrow 8 \rightarrow 0 \rightarrow 5$ as an example. Similarly, scenario 5 is both the original end and check-point. As it satisfies $5 \succ_{1} 0,5 \succ_{1} 12$, and $5 \succ_{2} 8$ and $5 \succ_{2} 20$, scenario 5 is the foresight incentive point and the route is foresight incentive route according to section 4.3. Therefore, scenario 20 is unstable for player 1 .

Take the route $5 \rightarrow 0 \rightarrow 8 \rightarrow 12 \rightarrow 52$ as an example. There is no $U M$ from scenario 52 for player 1 , thus scenario 5 is the original end. As $52 \prec_{2} 12,12 \prec_{1} 8,0$ $\prec_{2} 8$, scenario 8 is the check- point according to section 4.2. Furthermore, as $8 \prec_{1} 5$, there is no foresight incentive point in this route according to section 4.3.

According to Table 2., player 1 in scenario 5 can only move to scenario 0 by $U M$, but there are many routes starting with $5 \rightarrow 0$. As the aforementioned rules in section 4.3 , the necessary conditions of foresight incentive points in these routes are $S_{t} \succ_{1} 5$, and $S_{t} \succ_{2} 0$. Table 3. shows that this scenario does not exist. Thus, scenario 5 is stable for player 1. On the other hand, according to the foresight incentive algorithm, as scenario 5 is optimal for player 2 , it is impossible for him that the foresight incentive points exist in any routes starting with scenario 5 . Therefore, scenario 5 is stable for player 2. From the analysis above, scenario 5 is the only equilibrium obtained by the foresight incentive algorithm.

\section{Conclusions}

By applying "foresight incentive" to traditional conflict analysis, this paper has developed a foresight incentive algorithm, which can achieve more terse and stable equilibria. In addition, the stability of a practical conflict was analyzed, by applying both conflict analysis and foresight incentive algorithm. Through the case study, the effectiveness and superiority of the foresight incentive algorithm were verified. However, not all players in practice have enough foresight, and there may be more than two players in construction conflicts. These issues will be considered in future studies.

\section{References}

1) Howard, N.: Paradox of Rationality: Theory of Metagame and Political Behavior, The MIT Press, London, UK, 1971.

2) Fraser, N.M. and K.W. Hipel: Conflict Analysis: Models and Resolutions, North-Holland, New York, U.S.A., 1984.

3) Kilgour, D.M., K.W. Hipel and L. Fang: The graph model for conflicts, Automatica, Volume 23, Issue 1, 1987, pp.41-55.

4) Fang, L., K.W. Hipel, and D.M. Kilgour: Interactive decision making: the Graph Model for Conflict Resolution. New York: Wiley, 1993.

5) Howard, N.: The Management as Politician and General: The Metagame Approach to Analysing Cooperation and Conflict. In: Rosenhead, J. Editor, Rational analysis for a problematic world. Wiley, Chichester, UK, 1989, pp.239-261. 
6) Howard, N.: The CONAN Play: A Case Study Illustrating the Process of Metagame Analysis. In: Rosenhead, J. Editor, Rational analysis for a problematic world. Wiley, Chichester, UK, 1989, pp.263-283.

7) Okada, N., K.W. Hipel, N.M. Fraser and M. Fukushima: Conflictno-Suri, Gendai Sugakusha. Tokyo, Japan, 1988(in Japanese).

8) Ghanbarpour M.R. and K.W. Hipel: Sustainable development conflict over freeway construction, Environment, Development and Sustainability, 2009, Vol.11, No.2, pp.241-253.

9) Hipel, K.W., D.M. Kilgour, L. Fang, and X. Peng: The decision support system GMCR in environmental conflict management. Applied Mathematics and Computation, vol. 83, no.2-3, pp.117$152,1997$.

10) Amer, O., D.M. Kilgour and K.W. Hipel: Perceptual Graph Model Systems, Group Decision and Negotiation, 2009, Vol.18, No.3, pp.261-277.

11) Kilgour, D.M. and K.W. Hipel: The Graph Model for Conflict Resolution: Past, Present, and Future, 2005, No.6, pp.441-460.

12) Hua, F.: Foresight incentive algorithm and the development of the decision support system, [online]. Available from http://www. wanfangdata.com.cn/, 2010(in Chinese). 